\title{
THE RELEVANCE OF MICRORNAS IN VASCULAR AGING
}

\author{
Wong PF, Jong $\mathrm{HL}$ \\ Department of Pharmacology, Faculty of Medicine, University of Malaya, Kuala Lumpur
}

\author{
Correspondence: \\ Pooi-Fong Wong, \\ Department of Pharmacology, \\ Faculty of Medicine, University of Malaya, \\ 50603 Kuala Lumpur. \\ Tel: +603-7967 7022 (ext 2065); Fax: +603- 79674791 \\ E-mail:wongpf@um.edu.my
}

\begin{abstract}
MicroRNAs (miRNAs) are short, single-stranded non-coding RNAs that control gene expression by annealing to complementary sequences in mRNAs. They are estimated to regulate at least one third of human transcripts and hence, manipulation of these miRNAs can profoundly affect the proteome and ultimately cellular phenotypes. A substantial amount of work has shed light on the crucial roles of miRNAs in diseases. miRNA expression profiles between normal and diseased tissues have identified miRNA signature patterns that correlate to disease development and progression. This review discusses some of the important miRNAs that are involved in endothelial cell senescence and dysfunction that contribute to the development and progression of cardiovascular diseases.
\end{abstract}

Keywords: Cellular senescene, endothelial cells, miR-299-3p

\section{Introduction}

Cellular senescence is defined as the limited ability of cells to proliferate after a finite number of population doublings. Senescent cells do not divide but remain viable and metabolically active. These cells have enlarged and flattened morphology, increased granularity and vacuolization as well as adhesion to extracellular matrix while losing cell-to-cell contacts, altered patterns of gene and protein expression, and shortened telomere length. They characteristically express senescence-associated- $\beta$-galactosidase (SA- $\beta$-gal) activity at $\mathrm{pH} 6.0$ (1). Organismal aging is associated with cellular senescence as increased occurrence of senescent cells can be found in aged human tissues including the vasculature (2), skin (3), liver (4), kidney (5), astrocytes (6) and prostate (7), contributing to age-related diseases such as atherosclerosis, Alzheimer's disease and benign prostatic hyperplasia. Senescence can be triggered by the shortening of telomere during each cell cycle division which eventually leads to telomere "uncapping" which restricts cell division, hence, the term cellular senescence $(8,9)$. An imbalance in the biological redox reactions between the high level of oxidative stressors, such as hydrogen peroxide (10) and the low level of anti-oxidants can damage cellular DNA, RNA and proteins and induces cellular senescence (11), Other triggers of senescence include aberrant activation of oncogenes such as Ras and Myc, or silencing of tumor suppressor genes such as PTEN and $p R b$, can result in oncogenic stress and cause oncogene-induced senescence
(OIS) via three intrinsic pathways: p16/pRb-, Arf/p53/p21and DNA damage response (DDR)-pathway. Oncogeneinduced senescence often occur when the stress surpasses a threshold level, the cells are deprived of nutrients or growth factors, there are improper cell-cell contacts, and the cells are exposed to sub-lethal dose of DNA damaging agents such as anticancer drugs and gamma irradiation (8, 12). Cellular senescence has been described as a double edge sword since it does not only suppresses uncontrolled proliferation for cells at risk of neoplastic transformation (2), but also contributes to aged-related loss of tissue function or pathologies such as atherosclerosis in aged individuals (13).

MicroRNAs (miRNAs) are a well-recognized group of short non-coding RNAs that regulate gene expression by targeting 3' untranslated region (UTR) of mRNA transcripts and inhibit mRNA translation. In-depth details on the biogenesis and the mechanism of actions of miRNAs are reviewed at length elsewhere (14). Post-transcriptional regulation mediated by miRNA is pervasive in human because they are estimated to regulate at least one third of human transcripts as they can bind to multiple targets and suppress mRNAs translation (15). Although most miRNAs repress gene expression via translational inhibition or degradation of mRNA, some miRNAs can activate gene expression via upregulation of the translation of mRNA (16). Emerging evidence suggest that miRNAs play crucial roles in diverse biological processes including somatic 
and stem cell differentiation, cell proliferation, organ development, cell death, signaling in various diseases (17). Hence, it is not surprising that deregulation of miRNAs are associated with numerous diseases. These include cardiovascular diseases such as myocardial infarction and atherosclerosis (18), various types of cancer such as breast, lung, nasopharyngeal and thyroid carcinoma (1922), neurodegenerative diseases such as Alzheimer and Parkinson diseases $(23,24)$ and type 2 diabetes mellitus (25).

An important risk factor for cardiovascular diseases is aging (26). Accumulation of senescent cells in the vasculature leads to impaired vascular homeostasis. It has been reported that loss of vasomotor function of the major vasculature among elderly can contribute to the risk of development of cardiovascular diseases (27). It has been demonstrated that senescence of endothelial cells can lead to endothelial dysfunction due to an imbalance between vasodilating and vasoconstricting mediators released by (or acting on) the endothelium which subsequently results in reduced vasodilatation, a pro-thrombotic and pro-inflammatory state $(28,29)$. Endothelial dysfunction is further associated with atherosclerosis (30), hypertension (31), coronary artery disease (32), chronic heart failure (33) and peripheral artery disease (34). Previous studies have shown that deleterious changes in senescent endothelial cells lead to impairment of endothelium-dependent vasodilation and thrombogenesis that contribute to the pathogenesis of atherosclerosis (35). In addition, studies have shown that telomere shortening and activation of Notch pathway modulate senescence of endothelial cells in atherosclerosis (36).

In vitro studies using isolated human blood vessels and in vivo studies in animal and human demonstrated that there is a decrease in the release of endothelium-derived relaxing factors such as nitric oxide (NO), prostacyclin and endothelium-derived hyperpolarizing factor (EDHF) but an increase of endothelium-derived vasoconstrictors such as prostanoids and endothelin-1 (ET-1) in senescent endothelium (29). NO, the major relaxing factor is synthesized by endothelial nitric oxide synthase (eNOS) and the decline in NO level produced by senescent endothelium is reported to be due to a decline in the phosphorylation of eNOS by Akt in senescent endothelium (37). Impaired release of endothelium-derived relaxing factors leads to progressive endothelial dysfunction (29).

The importance of senescence in age-associated diseases highlights the need to study and further understand the molecular signaling of endothelial cell senescence, particularly on the role of miRNAs and their target genes in regulating senescence given that miRNAs are found deregulated in these diseases. This review discusses the involvement of selected well known miRNAs in endothelium senescence and vascular aging and their roles in cardiovacular diseases.

\section{miR-34a}

miR-34a is consistently upregulated in both cell culture and various organs of aged mouse (38-40). Overexpression of miR-34a has been shown to inhibit proliferation and to induce senescence of endothelial cells (39). The prime target of miR34a is Sirtuin 1 (SIRT1) (41) which is a $N A D^{+}$-dependent protein/histone deacetylase that regulates memory and plasticity, cell cycle progression, angiogenic functions, cellular senescence, apoptosis and various cellular metabolisms through its interaction with a number of molecules, including Ku70, p53, PML and FOXO (42-44). SIRT1 is essential for endothelial functions and it promotes longevity by preventing cellular senescence. Downregulation of SIRT1 by miR-34a is detrimental for endothelial and progenitor cells functions $(40,45)$. SIRT1 expression level decreased with age inversely to miR-34a in mouse brain tissues (39). More recently, miR-34a was found induced in aging mouse and human heart, and its silencing reduced age-associated cardiomyocyte cell death and improved myocardial function (46). This study further showed that silencing of miR-34a released the suppression of its other target, protein phosphatase 1 nuclear-targeting subunit (PNUTS), which reduces telomere shortening, DNA damage responses and cardiomyocyte apoptosis, and improves functional recovery after acute myocardial infarction (46). In coronary artery disease (CAD) patients, levels of miR-34a were also higher compared to the non-CAD group, whereas levels of SIRT1 protein were lower in the CAD group than in the non-CAD group. In patients treated with atorvastatin, a drug that lowers blood cholesterol, marked decrease in miR-34a levels and increase in SIRT1 levels were noted (45). Results from this study show that miR-34a is a valuable biomarker and should be explored for therapeutic monitoring of cardiovascular diseases and other age-related diseases.

\section{$\operatorname{miR}-217$}

The expression of miR-217 is upregulated in senescent endothelial cells and in human atherosclerotic plaques. Its expression level inversely correlates to those of SIRT1 and eNOS. Hence, overexpression of miR-217 downregulates SIRT1, increases eNOS acetylation and reduces eNOS protein expression leading to the reduction of the major endothelium relaxing factor, $\mathrm{NO}(38,47)$. miR-217 also reduces eNOS through the downregulation of FOXO1 (forkhead box 01) (38) and FOXO3 (48), which negatively regulates angiogenesis and vascular growth.

\section{miR-29 family members}

The miR-29 family members are upregulated in senescent endothelial cells (38), aortas of aged mice (49) and atrial tissues of dogs (50). The upregulation of the expression of miR-29 family members during senescence involves the 
activation of retinoblastoma tumor suppressor protein (Rb) pathway, which is a central pathway essential for the induction of senescence (51). Its overexpression is reported to be triggered by DNA damage and is mediated by $\mathrm{p} 53$ (52). Furthermore, miR-29 family members repressed Ppm1d phosphatase which in turn enhanced the activity of p53 (52). In normal aged ICR mouse and klotho-deficient senescence mouse models, the upregulation of miR-29 resulted in reduced type IV collagen, which can weaken the basal membrane of aged tissues (53). Using an aneurysm mouse model, it was shown that interference of miR-29 expression enhanced the expression of matrix protein and improved the integrity of the vascular wall, suggesting that miR-29 may be a valuable target for the maintenance of the vascular wall integrity (49).

\section{miR-146 family members}

Two members from this family, namely miR-146a and $-146 b$, are known for their roles in regulating inflammation. Their expressions are inducible by pro-inflammatory cytokines in cultured endothelial cells (54) and are found elevated in human atherosclerotic plaques (55). It was further shown that miR-146a and $-146 \mathrm{~b}$ suppressed the pro-inflammatory NF-KB pathway and downstream EGR and AP-1 transcription factors that drive inflammatory gene expression. This then suppressed endothelial activation and response to pro-inflammatory cytokines which in turn protected the vascular cells from cytokines-induced damages (54). Moreover, they also directly target and inhibit the expression of HuR, an RNA binding protein that promotes endothelial activation by increasing eNOS expression (54). By inhibiting HuR, endothelial activation can be suppressed. Other studies also revealed that miR146 family members control inflammation by inhibiting interleukin-1 receptor-associated kinase 1 (IRAK1), an enzyme required for the upregulation of NF-KB in fibroblast cells (56) and mouse heart tissues (57). Inhibition of IRAK1, hence, can inhibit the pro-inflammatory NF-KB pathway. miR-146a also targets NADPH oxidase 4 (NOX4) which is a predominant NOX in endothelial cells. NOX4 generates ROS and contributes to endothelial senescence and repression of NOX4 by miR-146, hence reduced oxidative stress and replicative senescence in endothelial cells (58). These studies collectively suggest that enhancement of miR-146 family members may be a potential strategy to protect vasculature against inflammation and oxidative stress-induced senescence and damages.

\section{miR-17-92 family}

miR-17-92 cluster is a well-defined group of miRNAs involved in endothelial functions and angiogenesis. This is a polycistronic miR cluster that encodes the miR-17, miR18a, miR-19a/b, miR-20a, and miR-92a. The miR-17-92 cluster is commonly linked to tumor angiogenesis, where overexpression of the miR-17-92 cluster promotes tumor angiogenesis (59). Specifically, miR-17, 18 and 19 exert their pro-angiogenic activities through the downregulation of the extracellular matrix proteins connective tissue growth factor (CTGF) and thrombospondin-1 (TSP-1), an anti-angiogenic molecule (60). This is also observed in aged mice, where the expressions of miR-18/19 concomitantly increased with the decreased in their targets, CTGF and TSP-1, which contributed to a failure-prone heart (61). On the other hand, miR-92a has anti-angiogenic activity where overexpression of miR-92a suppressed angiogenic sprout formation in vitro and interfered with intersegmental vessel growth in zebrafish (62). However, the role of each member in angiogenesis may be distinct in selective cells or environments. Combined overexpressions of miR-17, miR-18a, and miR-20a in endothelial cells can rescue the impaired endothelial functions caused by the inhibition of Dicer, an RNA endonuclease that regulates miR maturation (63). In contrast, overexpression of individual miR, namely, miR-17, miR-18a, miR-19a, and miR-20a alone, reduced sprouting of endothelial cells (64). It is unclear why their effects differed as a group and as individuals but the authors suggested that they have cell-intrinsic activities (64) and that they may also function differently in physiological and tumor angiogenesis (64). Hence, these data suggest that manipulation of these miRNAs for therapeutic purposes requires careful consideration since they are important modulators in both cancer and aging pathologies.

\section{miR-299-3p}

miRNAs discussed in this review represent some of the highly studied and consistently reported ones. As science advanced with more powerful and highly sensitive tools such as next generation sequencing and microRNA array system, it is not impossible to uncover novel miRNAs and to map their functions. We used an integrated miRNA and gene profiling approach to identify miRNAs and their targets that are associated with endothelial senescence (65). We found that miR-299-3p is up-regulated in senescent HUVECs compared to the young cells and one of its target genes could be IGF1, which is largely known to be associated with aging. Knockdown of miR-299-3p also resulted in significant reduction in the percentage of cells positively stained for senescence associated-bgalactosidase, increased in cell viability measured using MTT assay but marginal increases in cell proliferation and cell migration capacity measured by real-time growth kinetics analysis. Moreover, knockdown of hsa-miR-299$3 p$ also increased proliferation of $\mathrm{H}_{2} \mathrm{O}_{2}$-induced senescent endothelial cells. Our findings showed that knockdown of miR-299-3p can delay or protect against cellular senescence of endothelial cells by improving the metabolic activity of the senesced cells and this miRNA also acts in concert with miR-338-3p and miR-134 (65).

\section{Conclusion}

Evidence from various studies discussed above strongly support the notion that miRNAs are relevant and play 
important roles in endothelial cells senescence and vascular aging. While some of their target genes are known and some information on the associated mechanisms are defined, there remains much to be investigated. Prior to the development into miRNA-based therapeutics, many questions remain and these include the mechanism of increased or decreased expression of these miRNAs during senescence and aging, how and what regulate these miRNAs and whether their deregulation is direct effector of disease development or part of the feedback mechanisms. With the use of advanced technology such as next generation sequencing, more novel miRNAs are being identified and their functions validated. The answers to these questions will have significant implications to their use as therapeutics and biomarkers.

\section{Acknowledgement}

This study was supported by Fundamental Research Grant Scheme (FP042/2010A), Ministry of Education, Malaysia; University of Malaya Research Grant (RG097/09HTM) and Postgraduate Research Fund (PV044/2011B).

\section{References}

1. Campisi J, d’Adda di Fagagna F. Cellular senescence: when bad things happen to good cells. Nat Rev Mol Cell Biol 2007; 8:729-740.

2. Kumazaki T, Kobayashi M, Mitsui Y. Enhanced expression of fibronectin during in vivo cellular aging of human vascular endothelial cells and skin fibroblasts. Exp Cell Res 1993; 205:396-402.

3. Campisi J. The role of cellular senescence in skin aging. J Investig Dermatol Symp Proc 1998; 3:1-5.

4. Krizhanovsky V, Yon M, Dickins RA, Hearn S, Simon J, Miething $C$, Yee $H$, Zender L, Lowe SW. Senescence of activated stellate cells limits liver fibrosis Cell 2008; 134:657-667.

5. Melk A, Schmidt BM, Braun $H$, Vongwiwatana A, Urmson J, Zhu LF, Rayner D, Halloran PF. Effects of donor age and cell senescence on kidney allograft survival. Am J Transplant 2009; 9:114-123.

6. Bhat R, Crowe EP, Bitto A, Moh M, Katsetos CD, Garcia FU, Johnson FB, Trojanowski JQ, Sell C, Torres C. Astrocyte senescence as a component of Alzheimer's disease. PLoS One 2012; 7:e45069.

7. Castro P, Giri D, Lamb D, Ittmann M. Cellular senescence in the pathogenesis of benign prostatic hyperplasia. Prostate 2003; 55:30-38.

8. Ben-Porath I, Weinberg RA. The signals and pathways activating cellular senescence. Int J Biochem Cell Biol 2005; 37:961-976.

9. von Figura G, Hartmann D, Song Z, Rudolph KL. Role of telomere dysfunction in aging and its detection by biomarkers. J Mol Med (Berl) 2009; 87:1165-1171.

10. Higashi Y, Noma K, Yoshizumi M, Kihara Y. Endothelial function and oxidative stress in cardiovascular diseases. Circ J 2009; 73:411-418.
11. Sies H. Oxidative stress: oxidants and antioxidants. Exp Physiol 1997; 82:291-295.

12. Foreman KE, Tang J. Molecular mechanisms of replicative senescence in endothelial cells. Exp Gerontol 2003; 38:1251-1257.

13. Minamino T, Komuro I. Vascular cell senescence contribution to atherosclerosis. Circ Res 2007; 100:1526.

14. Finnegan EF, Pasquinelli AE. MicroRNA biogenesis: regulating the regulators. Crit Rev Biochem Mol Biol 2013; 48:51-68.

15. Lewis BP, Burge CB, Bartel DP. Conserved seed pairing, often flanked by adenosines, indicates that thousands of human genes are microRNA targets. Cell 2005; 120:15-20.

16. Vasudevan S, Tong Y, Steitz JA. Switching from repression to activation: microRNAs can up-regulate translation. Science 2007; 318:1931-1934.

17. Kloosterman WP, Plasterk RH. The diverse functions of microRNAs in animal development and disease. Dev Cell 2006; 11:441-450.

18. van Rooij E, Olson EN. MicroRNA therapeutics for cardiovascular disease: opportunities and obstacles. Nat Rev Drug Discov 2012; 11:860-872.

19. Liu N, Chen NY, Cui RX, et al. Prognostic value of a microRNA signature in nasopharyngeal carcinoma: a microRNA expression analysis. Lancet Oncol 2012; 13:633-641.

20. Mitomo S, Maesawa C, Ogasawara S, et al. Downregulation of miR-138 is associated with overexpression of human telomerase reverse transcriptase protein in human anaplastic thyroid carcinoma cell lines. Cancer Sci 2008; 99:280-286.

21. Piva R, Spandidos DA, Gambari R. From microRNA functions to microRNA therapeutics: Novel targets and novel drugs in breast cancer research and treatment (Review). Int J Oncol 2013; 43:985-994.

22. Yanaihara N, Caplen N, Bowman E, et al. Unique microRNA molecular profiles in lung cancer diagnosis and prognosis. Cancer Cell 2006; 9:189-198.

23. Maes OC, Chertkow HM, Wang E, et al. MicroRNA: Implications for Alzheimer Disease and other Human CNS Disorders. Curr Genomics 2009; 10:154-168.

24. Nunez-Iglesias J, Liu CC, Morgan TE, et al. Joint genome-wide profiling of miRNA and mRNA expression in Alzheimer's disease cortex reveals altered miRNA regulation. PLoS One 2010; 5:e8898.

25. Zampetaki A, Kiechl S, Drozdov I, et al. Plasma microRNA profiling reveals loss of endothelial miR126 and other microRNAs in type 2 diabetes. Circ Res 2010; 107:810-817.

26. North BJ, Sinclair DA. The intersection between aging and cardiovascular disease. Circ Res 2012; 110:10971108.

27. Versari D, Daghini E, Virdis A, et al. The ageing endothelium, cardiovascular risk and disease in man. Exp Physiol 2009; 94:317-321.

28. Endemann DH, Schiffrin EL. Endothelial Dysfunction. J Am Soc Nephrol 2004; 15:1983-1992. 
29. Vanhoutte PM. Ageing and endothelial dysfunction. Eur Heart J 2002; Suppl 4:A8-A17.

30. Hayashi T, Matsui-Hirai $H$, Miyazaki-Akita A, et al. Endothelial cellular senescence is inhibited by nitric oxide: Implications in atherosclerosis associated with menopause and diabetes. Proc Natl Acad Sci U S A 2006; 103:17018-17023.

31. Sainani GS, Maru VG. Role of endothelial cell dysfunction in essential hypertension. J Assoc Physicians India 2004; 52:966-969.

32. Abrams J. Role of endothelial dysfunction in coronary artery disease. Am J Cardiol 1997; 79:2-9.

33. Bauersachs J, Widder JD. Endothelial dysfunction in heart failure. Pharmacol Rep 2008; 60:119-126.

34. Brevetti G, Silvestro A, Di Giacomo S, et al. Endothelial dysfunction in peripheral arterial disease is related to increase in plasma markers of inflammation and severity of peripheral circulatory impairment but not to classic risk factors and atherosclerotic burden. J Vasc Surg 2003; 38:374-379.

35. Davignon J, Ganz P. Role of endothelial dysfunction in atherosclerosis. Circulation 2004; 109:III27-32.

36. Liu ZJ, Tan Y, Beecham GW, et al. Notch activation induces endothelial cell senescence and proinflammatory response: implication of Notch signaling in atherosclerosis. Atherosclerosis 2012; 225:296-303.

37. Smith AR, Hagen TM. Vascular endothelial dysfunction in aging: loss of Akt-dependent endothelial nitric oxide synthase phosphorylation and partial restoration by (R)- $\alpha$-lipoic acid. Mol Mech of Signaling 2003; 31:1447-1449.

38. Olivieri F, Rippo MR, Monsurro V, et al. MicroRNAs linking inflamm-aging, cellular senescence and cancer. Ageing Res Rev 2013; 12(4): 1056-1068.

39. Li X, Khanna A, Li N, Wang E. Circulatory miR34a as an RNAbased, noninvasive biomarker for brain aging. Aging (Albany NY) 2011; 3:985-1002.

40. Ito T, Yagi S, Yamakuchi M. MicroRNA-34a regulation of endothelial senescence. Biochem Biophys Res Commun 2010; 398:735-740.

41. Yamakuchi M, Lowenstein CJ. MiR-34, SIRT1 and p53: the feedback loop. Cell Cycle 2009; 8:712-715.

42. Cohen HY, Lavu S, Bitterman KJ, et al. Acetylation of the $\mathrm{C}$ terminus of Ku70 by CBP and PCAF controls Bax-mediated apoptosis. Mol Cell 2004; 13:627-638.

43. Langley $E$, Pearson $M$, Faretta $M$, et al. Human SIR2 deacetylates p53 and antagonizes PML/p53-induced cellular senescence. EMBO J 2002; 21:2383-2396.

44. Brunet A, Sweeney LB, Sturgill JF, et al. Stressdependent regulation of FOXO transcription factors by the SIRT1 deacetylase. Science 2004; 303:2011-2015.

45. Tabuchi $T$, Satoh $M$, ItohT, et al. MicroRNA-34a regulates the longevity-associated protein SIRT1 in coronary artery disease: effect of statins on SIRT1 and microRNA-34a expression. Clin Sci (Lond) 2012; 123:161-171.

46. Boon RA, lekushi K, Lechner S, Seeger T, Fischer A, Heydt S, Kaluza D, Tréguer K, Carmona G, Bonauer A, Horrevoets AJ, Didier N, Girmatsion Z, Biliczki P,
Ehrlich JR, Katus HA, Müller OJ, Potente M, Zeiher AM, Hermeking H, Dimmeler S. MicroRNA-34a regulates cardiac ageing and function. Nature 2013; 495:107-110.

47. Menghini R, Casagrande V, Cardellini $M$, et al. MicroRNA 217 Modulates Endothelial Cell Senescence via Silent Information Regulator 1. Circulation 2009; 120:1524-U1102.

48. Zhang S, Liu L, Wang R, Tuo H, Guo Y, Yi L, Wang D, Wang J. MicroRNA-217 Promotes Angiogenesis of Human Cytomegalovirus-Infected Endothelial Cells through Downregulation of SIRT1 and FOXO3A. PLOS One 2013; 8:e83620.

49. Boon RA, Seeger T, Heydt S, et al. MicroRNA-29 in aortic dilation: implications for aneurysm formation. Circ Res 2011; 109:1115-1119.

50. Xu GJ, Gan TY, Tang BP, Chen ZH, Ailiman M, Zhou $X H$, Jiang T, Song JG, Guo X, Li YD, Miao HJ, Zhang Y, $\mathrm{Li} J X$. Changes in microRNAs expression are involved in age-related atrial structural remodeling and atrial fibrillation. Chin Med J (Engl) 2013; 126:1458-1463.

51. Martinez I, Cazalla D, Almstead LL, Steitz JA, DiMaio D. miR-29 and miR-30 regulate B-Myb expression during cellular senescence. Proc Natl Acad Sci U S A. 2011; 108(2):522-527.

52. Ugalde AP, Ramsay AJ, de la Rosa J, Varela I, Mariño G, Cadiñanos J, Lu J, Freije JM, López-Otín C. Aging and chronic DNA damage response activate a regulatory pathway involving miR-29 and p53. EMBO J 2011; 30:2219-2232.

53. Takahashi M, Eda A, Fukushima T, Hohjoh H. Reduction of type IV collagen by upregulated miR-29 in normal elderly mouse and klotho-deficient, senescencemodel mouse. PLoS One 2012; 7:e48974.

54. Cheng HS, Sivachandran N, Lau A, Boudreau E, Zhao $\mathrm{JL}$, Baltimore D, Delgado-Olguin P, Cybulsky MI, Fish JE. MicroRNA-146 represses endothelial activation by inhibiting pro-inflammatory pathways. EMBO Mol Med 2013; 5:949-966.

55. Raitoharju E, Lyytikäinen LP, Levula M, Oksala N, Mennander A, Tarkka M, Klopp N, Illig T, Kähönen M, Karhunen PJ, Laaksonen R, Lehtimäki T. miR-21, miR-210, miR-34a, and miR-146a/b are up-regulated in human atherosclerotic plaques in the Tampere Vascular Study. Atherosclerosis 2011; 219:211-217.

56. Xie YF, Shu R, Jiang SY, Liu DL, Ni J, Zhang XL. MicroRNA-146 inhibits pro-inflammatory cytokine secretion through IL-1 receptor-associated kinase 1 in human gingival fibroblasts. J Inflamm (Lond) 2013; 10:20.

57. Chassin C, Hempel C, Stockinger S, Dupont A, Kübler JF, Wedemeyer J, Vandewalle A, Hornef MW. MicroRNA146a-mediated downregulation of IRAK1 protects mouse and human small intestine against ischemia/ reperfusion injury. EMBO Mol Med 2012; 4:1308-1319.

58. Vasa-Nicotera $M$, Chen $H$, Tucci $P$, et al. miR-146a is modulated in human endothelial cell with aging. Atherosclerosis 2011, 217:326-330.

59. Dews M, Homayouni A, Yu D, Murphy D, Sevignani C, Wentzel E, Furth EE, Lee WM, Enders GH, Mendell JT, Thomas-Tikhonenko A. Augmentation of tumor 
angiogenesis by a Myc-activated microRNA cluster. Nat Genet 2006; 38:1060-1065.

60. Kuhnert F, Kuo CJ. miR-17-92 angiogenesis micromanagement. Blood. 2010; 115(23):4631-4633.

61. van Almen GC, Verhesen $W$, van Leeuwen $R E$, van de Vrie M, Eurlings C, Schellings MW, Swinnen M, Cleutjens JP, van Zandvoort MA, Heymans S, Schroen B. MicroRNA-18 and microRNA-19 regulate CTGF and TSP-1 expression in age-related heart failure. Aging Cell 2011; 10:769-779.

62. Bonauer A, Carmona G, Iwasaki M, Mione M, Koyanagi M, Fischer A, Burchfield J, Fox H, Doebele C, Ohtani K, Chavakis E, Potente M, Tjwa M, Urbich C, Zeiher AM,
Dimmeler S. MicroRNA-92a controls angiogenesis and functional recovery of ischemic tissues in mice. Science 2009; 324:1710-1713.

63. Suarez Y, Fernandez-Hernando C, Yu J, et al. Dicerdependent endothelial microRNAs are necessary for postnatal angiogenesis. Proc Natl Acad Sci U S A 2008; 105:14082-14087.

64. Doebele C, Bonauer A, Fischer A, et al. Members of the microRNA-17-92 cluster exhibit a cell-intrinsic antiangiogenic function in endothelial cells. Blood 2010; 115:4944-4950.

65. Jong HL, Mustafa MR, Vanhoutte PM, et al. MicroRNA 299-3p modulates replicative senescence in endothelial cells. Physiol Genomics 2013; 45:256-267. 